\title{
Effect of detrusor overactivity on the expression of aquaporins and nitric oxide synthase in rat urinary bladder following bladder outlet obstruction
}

\author{
Sun-Ouck Kim, MD; 'Dongjune Choi, MD; ; Seung Hee Song, MD; Kyu Youn Ahn, MD; Dongdeuk Kwon, MD;* \\ Kwangsung Park, MD; Soo Bang Ryu, MD*
}

*Department of Urology, Chonnam National University Medical School, Gwangiu, Korea; †Department of Anatomy, Chonnam National University Medical School, Gwangiu, Korea

Cite as: Can Urol Assoc J 2013;7(5-6):e268-74. http://dx.doi.org/10.5489/cuaj.993 Published online May 13, 2013.

\section{Abstract}

Background: Aquaporins (AQPs) have recently been reported to be expressed in rat and human urothelium. Nitric oxide (NO) is thought to play a role in the bladder overactivity related to bladder outlet obstruction (BOO). The purpose of this study is to investigate the effect of $\mathrm{BOO}$ on the expression of AQP2-3 and nitric oxide synthase (NOS) isoforms in rat urothelium.

Methods: Female Sprague-Dawley rats $(230-240 \mathrm{~g}, \mathrm{n}=60)$ were divided into 2 groups. The control group $(\mathrm{n}=30)$ and the partial bladder outlet obstruction (BOO) group $(n=30)$. After 4 weeks, we performed a urodynamic study to measure the contraction interval and contraction pressure. The expression and cellular localization of AQP2-3, endothelial nitric oxide synthase (eNOS) and neuronal nitric oxide synthase ( $\mathrm{nNOS}$ ) were determined by Western blot and immunohistochemistry.

Results: On the cystometrogram, the estimated contraction interval time (minutes, mean $\pm \mathrm{SE}$ ) was significantly lower in the $\mathrm{BOO}$ group $(3.0 \pm 0.9)$ than in the control group $(6.3 \pm 0.4 ; p<0.05)$. AQP2 was localized in the cytoplasm of the epithelium, whereas AQP3 was found only in the cell membrane of the epithelium. The protein expression of AQP2-3, eNOS and nNOS was significantly increased in the BOO group.

Conclusion: Detrusor overactivity induced by $\mathrm{BOO}$ causes a significant increase in the expression of AQP2-3, eNOS, and nNOS in rat urinary bladder. This may imply that the AQPs and NOS isoforms have a functional role in the bladder dysfunction that occurs in association with BOO.

\section{Introduction}

Despite the high prevalence of bladder outlet obstruction (BOO) among older men, the underlying mechanisms responsible for the bladder dysfunction induced by $\mathrm{BOO}$ remain poorly understood. In the past, the urothelium was considered a simple barrier between the urine and the circulating blood system. The urothelium is now understood to be an active organ that plays an important role in regulating bladder disorders. ${ }^{1,2}$ It has been suggested that the urothelium can mediate water and solute transport under certain circumstances. ${ }^{3-5}$ Several studies have shown that the urinary bladder epithelium contains a sodium transporter ${ }^{6}$ that has been identified as an epithelial sodium channel (ENaC), ${ }^{7}$ which is known to be responsible for salt and fluid transport across the epithelia of many tissues. ${ }^{8}$

Aquaporins (AQPs) are a family of intrinsic membrane proteins that function as water channels in many cell types involved in fluid transport. ${ }^{9}$ Functional measurements indicate that AQPs transport not only water, but also urea, glycerol and other small solutes. These aquaporins are called aquaglyceroporins. ${ }^{10}$ Until now, however, only limited data have been available on the role of AQPs in the mammalian urinary bladder. Recently, Spector and colleagues investigated the regulation of AQPS in the urothelium of rats following a dehydration test. ${ }^{11}$ They suggested that the AQPS in the urothelium may play a role in epithelial cell volume and osmolarity regulation and introduced the possibility of water movement across the urothelium. Rubenwolf and colleagues revealed that AQPs are expressed in cultured human urothelium, suggesting a potential role in transurothelial water and solute transport. ${ }^{12}$ Recently, we reported AQP1 expression in the capillaries and venules of the urinary bladder and significant incease of protein expression of AQP1 in the BOO rat urinary bladder. ${ }^{13}$ No studies to date have investigated the expression of AQP2-3 in the urinary bladder of rats after $\mathrm{BOO}$ or the functional activity of these proteins in response to $\mathrm{BOO}$.

Recent reports dealing with $A Q P s$, nitric oxide synthase (NOS isoforms) and water permeability show that an NO-mediated pathway results in the insertion of AQP2 from the cytoplasm into the plasma membrane in the kidney. ${ }^{14,15}$ It has been suggested that NO could modify plasma membrane proteins, such as AQP1, and interfere with their function. Also, NO is thought to play a role in bladder overactivity, which may be associated with impairment in the NO 
signaling pathway. ${ }^{16,17}$ On the basis of these findings, we hypothesized that the AQPs and NOS might be involved in the physiology of bladder dysfunction induced by BOO. The purpose of this study was to investigate the impact of $\mathrm{BOO}$ on the expression of the AQP2-3 and NOS isoforms in rat urinary bladder.

\section{Methods}

\section{Experimental model}

Female Sprague-Dawley rats $(230-240 \mathrm{~g}, \mathrm{n}=60)$ were divided into 2 groups: control (Con group, $\mathrm{n}=30$ ) and bladder outlet obstruction (BOO group, $n=30$ ). The BOO group underwent partial BOO. Animals were premedicated with xylazine $(2.2 \mathrm{mg} / \mathrm{kg}, \mathrm{IM})$ and were anesthetized with a zolazepam/tiletamine cocktail $(4.4 \mathrm{mg} / \mathrm{kg}$, IM). A midline abdominal incision was made, and the bladder and proximal urethra were dissected free of the surrounding tissue. To create an intravesical obstruction, we placed a PE-90 (polyethylene catheter-90) catheter beside the proximal urethra and tied a 3-zero silk ligature around the urethra and catheter. The catheter was subsequently removed and the abdominal incision was closed. The control group underwent a sham operation. After 4 weeks, urodynamic and histologic studies were done. All rats were deprived of food overnight before the experiments were performed; before that time, they were given a standard diet. The protocol for animal surgery was approved by the Ethics Committee of the Chonnam National University Medical School.

\section{Cystometrograms}

Rats were anesthetized with $1.2 \mathrm{~g} / \mathrm{kg}$ urethane injected subcutaneously. A suprapubic midline incision was performed to expose the bladder, a transvesical catheter with a fireflared tip (PE-50) was inserted into the dome of the bladder and secured with a ligature, and the abdomen was closed. The catheter was connected to a pressure transducer and syringe pump via a 3-way stopcock to record intravesical pressure and to infuse saline into the bladder. After the bladder was emptied, cystometry was performed with saline infused at a rate of $0.04 \mathrm{ml} / \mathrm{min}$. The contraction pressure and contraction interval were recorded.

\section{Western blot}

The tissue homogenates ( $30 \mu \mathrm{g}$ of protein) were separated by $12 \%$ SDS-polyacrylamide gel electrophoresis and transferred to PVDF membranes (Amersham Pharmacia Biotech, England, UK). The blots were then washed with Tris-buffered saline Tween-20 (TBST) $(10$ mM Tris-HCl, pH
7.6, $150 \mathrm{mM} \mathrm{NaCl}, 0.05 \%$ Tween-20). The membrane was blocked with 5\% skimmed milk for 1 hour and incubated with the appropriate primary antibody. Monoclonal rabbit antibodies for AQP2-3 (Chemicon, Temecula, CA; 1:2000, $1: 1000$, and 1:1000, respectively), eNOS and nNOS (Santa Cruz, CA; 1:1000 and 1:1000, respectively), and glyceraldehyde 3-phosphate dehydrogenase (GAPDH) (Sigma, St. Louis, MO; 1:1000) were used. The membrane was then washed and detected with goat anti-rabbit-lgG conjugated to horseradish peroxidase. Antibody incubations were performed in a $4^{\circ} \mathrm{C}$ incubator. The bands were visualized by using enhanced chemiluminescence (Amersham Pharmacia Biotech, England, UK).

\section{Immunohistochemistry}

The bladder tissue was dissected from both lateral walls of the bladder. The tissue was placed in $4 \%$ paraformaldehyde fixative for 16 hours and then processed for washing and dehydration. The tissue was routinely embedded in paraffin, and $6-\mu \mathrm{m}$ sections were prepared. Tissues were stained with hematoxylin and eosin (H\&E) stain. Immunohistochemistry was performed by using an immunoperoxidase procedure (Vector ABC Kit; Vector Laboratories, Burlingame, CA). The tissue sections were deparaffinized in xylene, dehydrated in a graded series of ethanol, rinsed twice in phosphatebuffered saline (PBS), and then treated with $3 \% \mathrm{H}_{2} \mathrm{O}_{2}$ in $60 \%$ methanol for 30 minutes to quench endogenous peroxidase activity. After washing twice (5 minutes) in PBS, the sections were incubated for 12 to 14 hours with purified rabbit antibodies for AQP2-3 (Chemicon, Temecula, CA; 1:2000, 1:1000, 1:1000, respectively) and monoclonal rabbit antibodies against eNOS and nNOS (Santa Cruz, CA; $1: 1000$ and $1: 1000$, respectively) in PBS with $0.3 \%$ bovine serum albumin. In the negative control, the sections were incubated in PBS containing 5\% normal goat serum only. The sections were then rinsed 3 times in PBS and incubated sequentially for 30 minutes, each with the biotinylated secondary antibody and the $A B C$ reagent. The sections were then incubated for 5 minutes with the peroxidase substrate solution contained in the kit.

\section{Statistical analysis}

The results are expressed as the mean \pm the standard error (SE). The Mann-Whitney test was performed for statistical analysis. Differences were considered significant at $p<0.05$.

\section{Results}

There was no significant difference in body weight between the groups. The weights ( $\mathrm{mg}$ ) of the bladders, however, were significantly $(p<0.05)$ higher in the BOO group $(643 \pm 18.9)$ 
Kim et al.

than in the control group $(114.2 \pm 18.3)$, thus supporting the proper creation of $\mathrm{BOO}$.

\section{Cystometric changes in $\mathrm{BOO}$}

In the cystometrograms, the average contraction pressures $(\mathrm{mm} \mathrm{Hg})$ were significantly higher $(p<0.05)$ in the BOO group $(19.6 \pm 4.2)$ than in the control group $(12.6 \pm 3.6)$ (Fig. 1). The contraction interval ( $\mathrm{min})$, however, was significantly lower $(p<0.05)$ in the BOO group $(3.0 \pm 0.9)$ than in the control group (6.3 \pm 0.4$)$ (Fig. 1).
Effect of BOO on the expression of AQP 2-3

The proportion of bladder smooth muscle was markedly increased in the BOO group compared with the control (Fig. 2). AQP2 was expressed throughout the cytoplasm of the epithelium and detrusor muscle layer, whereas AQP3 was expressed throughout the cell membrane of the urothelium and not in the cell cytoplasm (Fig. 3). Immunohistochemistry showed a tendency for increased expression of AQP2-3 in the $\mathrm{BOO}$ group compared with the control (Fig. 3). Western blot analysis revealed bands for AQP protein at $28 \mathrm{kDa}$ (Fig. 4, part a). AQP2-3 protein was recognized in all groups, but there was a significant increase in the $\mathrm{BOO}$ group compared with the control $(p<0.05)$ (Fig. 4, part a).
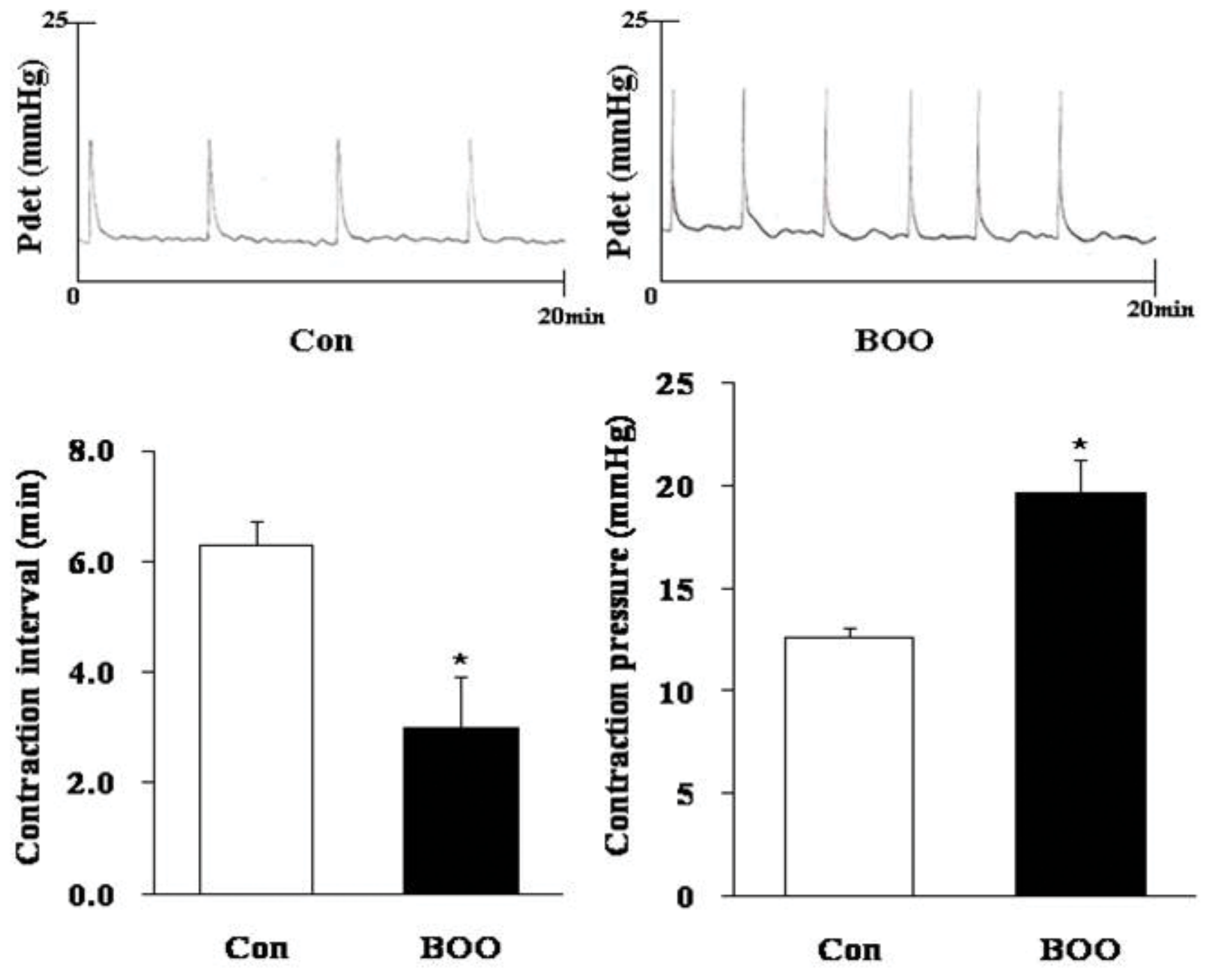

Fig. 1. Representative urodynamic profiles of control and bladder outlet obstruction (BOO) bladders. In the control group, voiding contractions were observed about every 5 minutes. The contraction interval was decreased significantly in the B00 group compared with the control $\left(p<0.05^{*}\right)$. Note the increased peak pressures on each voiding contraction in the BO0 group. The lower panels denote the means \pm SE of 10 experiments for each condition. ${ }^{*} p<0.05$ vs. control. Con: control; B00: bladder outlet obstruction. 


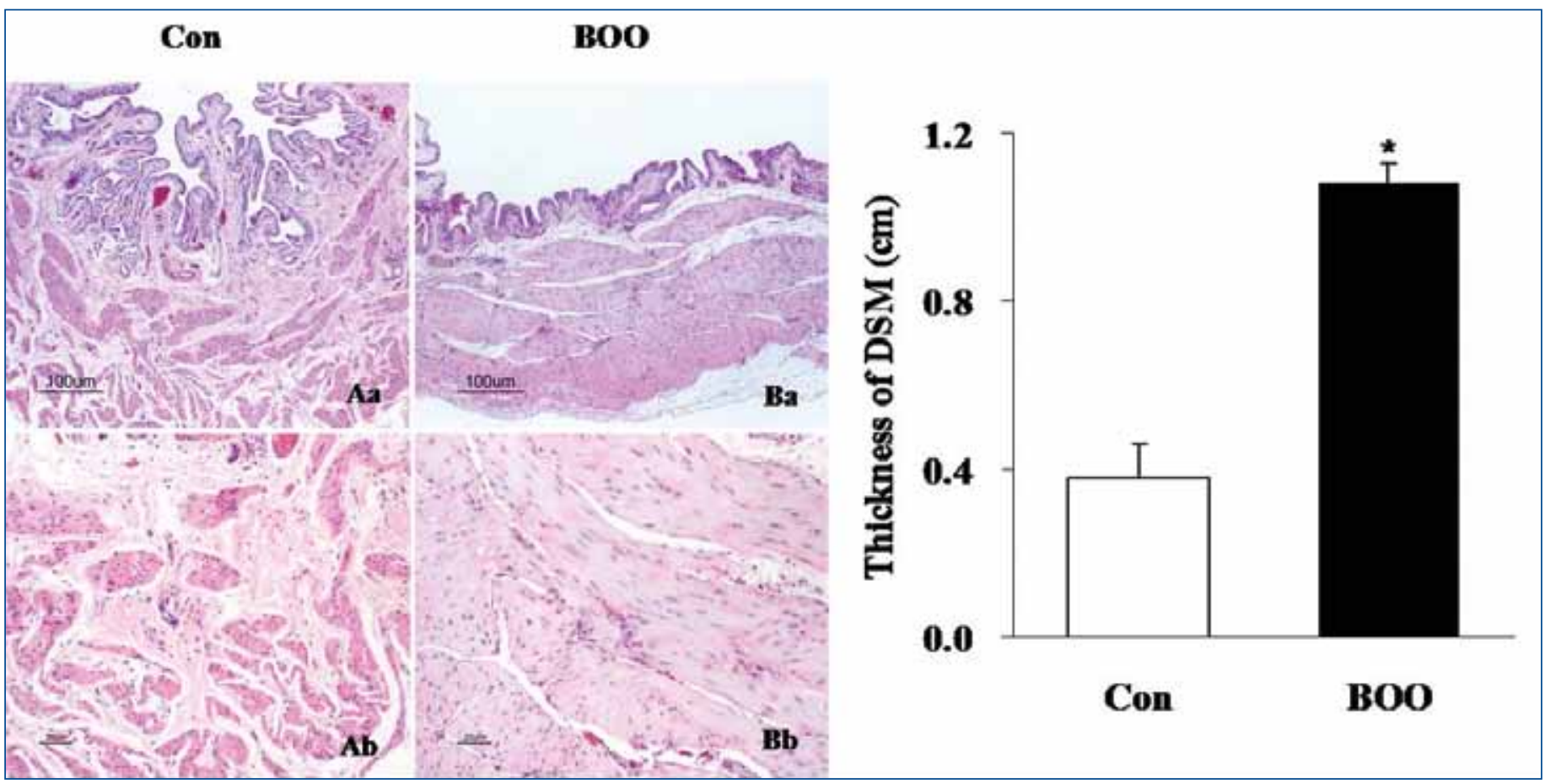

Fig. 2. Hematoxylin and eosin (H\&E) staining of the urinary bladder tissue in rats of the control (Con) and bladder outlet obstruction (B00) groups. Smooth muscle content $(B a, B b)$ was significantly increased in the B00 group compared with the control group $(A a, A b)$. Ab and $B b$ is the magnification of the detrusor muscle from each $\mathrm{Aa}$ and $\mathrm{Ba}$. The horizontal scale bar at the bottom of each figure indicates the magnification power. Con: control; B00: bladder outlet obstruction.

\section{Effect of BOO on the expression of e-NOS and n-NOS}

Western blot analysis revealed bands at $140 \mathrm{kDa}$ for eNOS protein and at $155 \mathrm{kDa}$ for $\mathrm{nNOS}$ protein (Fig. 4, part b). NOS protein expression was significantly increased in the BOO group compared with the control $(p<0.05)$ (Fig. 4, part b).

\section{Discussion}

In the current study, we showed that the cystometric parameters of contraction pressure and micturition interval were significantly changed in the $\mathrm{BOO}$ rats. The expression of AQP2-3 and NOS was also significantly increased in the $\mathrm{BOO}$ group. These results provide evidence that $\mathrm{BOO}$ induces bladder overactivity, and that the AQPs and NOS isoforms may play a role in the bladder dysfunction induced by BOO. To our knowledge, this study is the first to shed some light on the possible occurrence of water and solute movement via AQPs in the urinary bladder epithelium in the detrusor overactivity induced by BOO.

New ideas and evidence have emerged regarding the role of the urothelium in the physiology of the urinary bladder. It has generally been assumed that the bladder is a simple organ for the storage and emptying of urine. Now, it is understood that the urothelium plays an active role in the regulation of the bladder. Increased awareness that the urothelium has a significant transport function ${ }^{18}$ has led investigators to question whether the urothelium has a role in water and solute movement from the urine to the systemic circulation. The composition of the urine has been found to change during its transport through urine passages, a finding ascribed to a modifying function of the urothelium. ${ }^{19,20}$ Indeed, the mammalian urothelium modifies the urine concentration and composition of solutes by reabsorbing $\mathrm{K}^{+}$, urea, and creatine and secreting $\mathrm{Na}^{+} \cdot{ }^{20,21}$ Furthermore, a urothelial $\mathrm{Na}^{+}$transporter has recently emerged; its proposed role involves water and salt transport and sensing bladder fullness. ${ }^{17,21}$ Apical $\mathrm{Na}^{+}$transport is mediated by $\mathrm{Na}^{+}$channels that are sensitive to changes of luminal hydrostatic pressure and osmolarity. ${ }^{22,23}$

In humans, lower voided volumes are associated with low urinary $\mathrm{pH}$, high urinary $\mathrm{Na}^{+}$and $\mathrm{K}^{+}$, and osmolarity. ${ }^{19,24}$ These effects could be exacerbated if the urothelium were damaged and may also give rise to bladder pain and increased urinary frequency. ${ }^{18}$ This possibility may be further supported by the results of Araki and colleagues. ${ }^{25}$ They studied the role of the $\mathrm{ENaC}$ in bladder dysfunction in male patients who were diagnosed clinically with BOO. They reported that the expression levels of $\mathrm{ENaC}$ were significantly greater in patients with $\mathrm{BOO}$ than in controls and correlated with the patients' storage symptom scores. They insisted that this expression in the bladder epithelium might be implicated in the mechanosensory transduction in the 


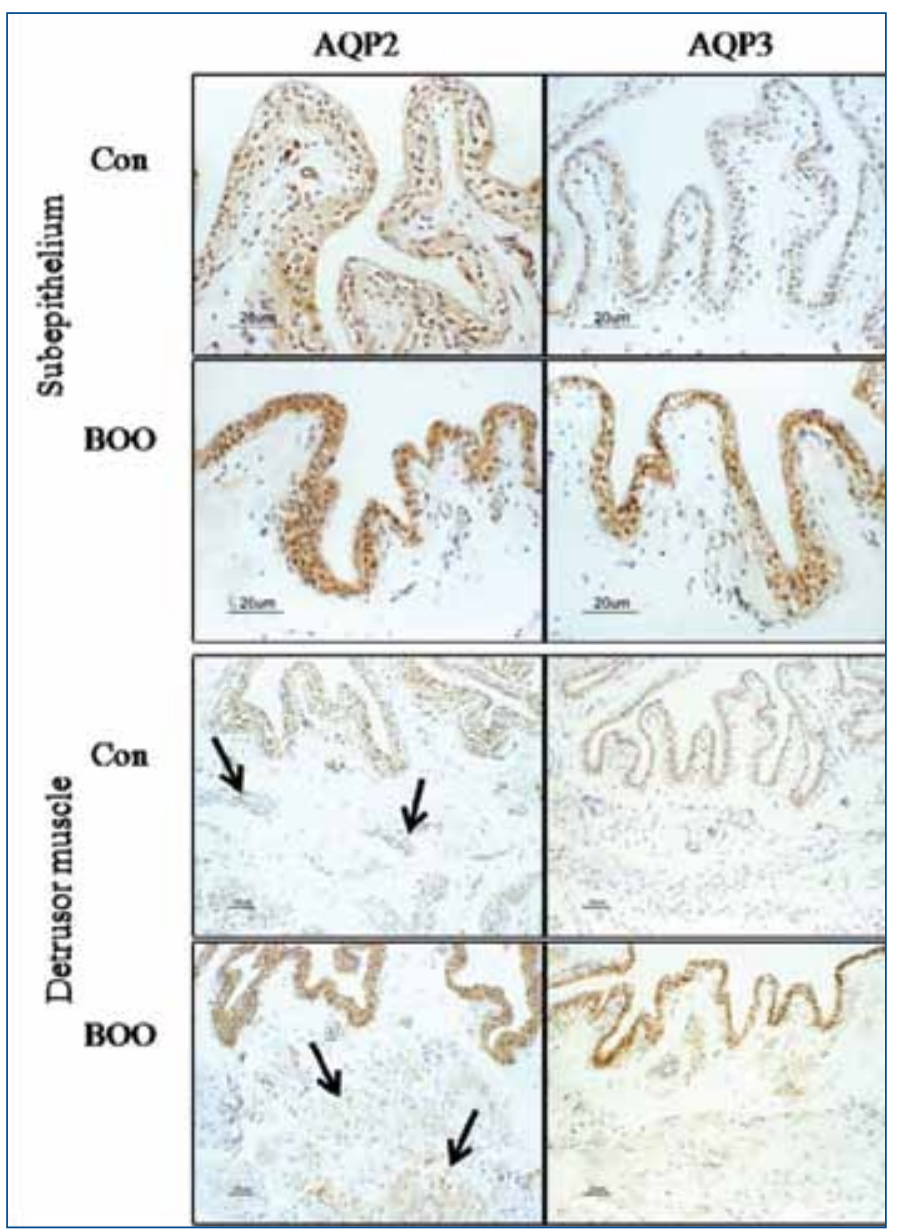

Fig. 3. Immunohistochemistry of AQP2-3 of rat urinary bladder tissue in the control (Con) and bladder outlet obstruction (BOO) groups. AQPs are stained brown via immunolabeling. AQP2 was expressed throughout the cytoplasm of the epithelium and detrusor muscle layer (arrows). AQP3 was expressed in the cell membrane of the epithelium. In the BOO group, the tendency of increased expression of AQP2-3 was observed in the BOO group compared with the control. The horizontal scale bar at the bottom of each figure indicates the magnification power. Con: control; BOO: bladder outlet obstruction.

bladder afferent pathways that induce detrusor overactivity as a result of $\mathrm{BOO}$.

AQPs are expressed in many fluid-containing tissues and are involved in many physiological mechanisms, including the transport of transepithelial fluid, the concentration of urine and gland fluid secretion. ${ }^{26}$ Until now, however, limited data have been available concerning AQPs in the urinary bladder. AQP1 was reported to be selectively expressed in the microvasculature, and AQP2 and AQP3 are commonly expressed in epithelial cells in various organs. ${ }^{26}$ Therefore, we investigated the expression of AQPs with a special focus on AQP1-3 in the urinary bladder to support the hypothesis of water movement in the bladder from the subepithelial capillaries to the urothelium. In our previous study, we reported that AQP1 was mainly expressed in the capillaries and venules of the urinary bladder and that sig- nificantly increased protein expression of AQP1 in the BOO rat urinary bladder..$^{13}$ In the current study, immunolabeling of AQP2 was expressed throughout the epithelial cell layer and detrusor smooth muscle, and AQP3 was expressed only throughout the epithelial cell layer.

The water transport mechanism by AQPs may be influenced by the NOS system. Many studies have shown that AQPs are important for concentrating urine in the kidneys. NOS has been implicated in modulating renal blood flow and in the regulation of sodium handling. ${ }^{27}$ Recently, Bouley and colleagues ${ }^{14}$ cited a close relationship between $\mathrm{NO}$ and AQP2 and suggested that NO stimulates the membrane insertion of AQP2 in renal epithelial cells. They suggested that the phosphorylation of AQP2 was required for signaling of AQP2 to shuttle from an intracellular vesicle to the plasma membrane. Similarly, it is postulated that during bladder filling, the urothelium undergoes numerous changes, including increases in the surface area of umbrella cells and movement of cytoplasmic discoid vesicles to the plasma membrane. ${ }^{28}$ The trafficking of intracellular vesicles to the plasma membrane in response to mechanical force is also associated with the release of neurotransmitters, such as NO and ATP, which may act on submucosal afferent sensory nerve fibers.

In the present study, the expression of AQP2-3 was significantly affected by $\mathrm{BOO}$, which suggests that AQPs are influenced by the bladder dysfunction induced by anatomical changes, such as BOO. This agrees with a previous study of AQP expression in rat urinary bladder. ${ }^{11}$ In this previous study by Spector and colleagues, the authors showed that dehydration in rats led to a significant upregulation of AQP2 and AQP3 expression, thus providing presumptive evidence that AQPs are involved in water and solute transport. Rubenwolf and colleagues ${ }^{12}$ demonstrated the expression of AQPs in human urothelium and suggested a potential role in transurothelial water and solute transport in human bladder. One of the possible reasons behind this influence on AQP expression is the significance of the location of AQP expression. Epithelial changes in the microvasculature (AQP1) and urothelium (AQP2, AP3) and detrusor muscle hypertrophic changes (AQP2) happen after BOO. Our results demonstrated that $\mathrm{BOO}$ led to significant upregulation of AQP2-3 expression, providing presumptive evidence that the AQPs are involved in the increased bladder activity induced by $\mathrm{BOO}$, probably via modification of urothelial water and solute composition. It could be suggested that $\mathrm{BOO}$ may activate the NOS system, which regulates AQP1 in capillaries and allows plasma transudation from the capillaries to the subepithelial layer. Then, in the epithelial layer, AQP2 and AQP3 may open water channels and result in transepithelial fluid and solute being transported to either side.

A limitation of this study is that the precise functional relationship between the AQPs and the NOS isoforms has not yet been fully unveiled, although we did show the expres- 


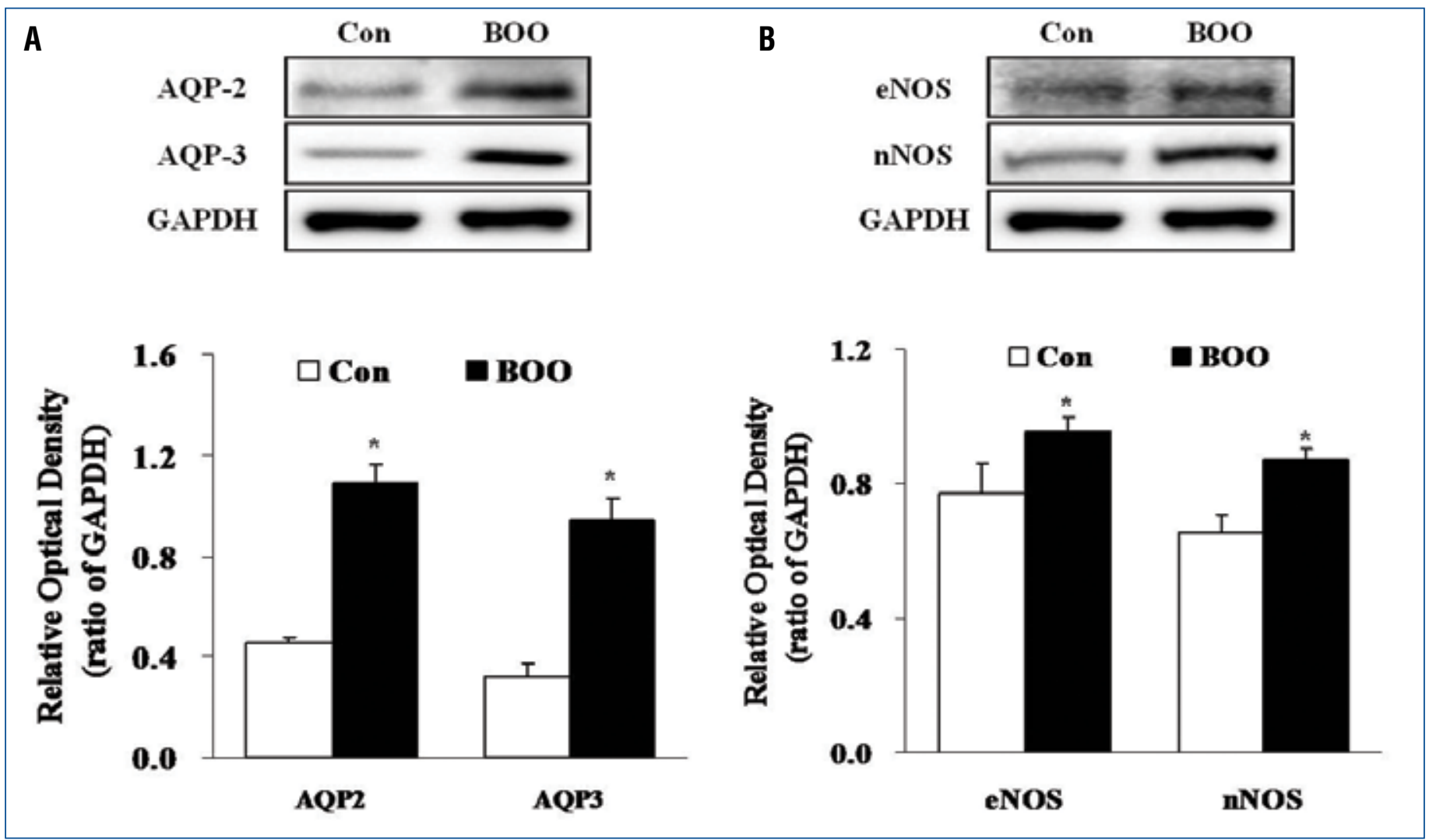

Fig. 4. Immunoblotting of AQP2-3, eNS and nNOS in the rat urinary bladder. (A) AQP2-3 protein expression was significantly increased in the B00 group. The antiAQP antibodies recognize 27 to $29 \mathrm{kDa}$ bands corresponding to glycosylated AQPs. The lower panels denote the means \pm SE of 10 experiments for each condition determined by densitometry relative to GAPDH.* (B) eNOS and nNOS protein expression was significantly increased in the BOO group. The protein bands at approximately $140 \mathrm{kDa}$ represent the eNOS protein and those at $155 \mathrm{kDa}$ represent the nNOS protein. The lower panels denote the means \pm SE of 10 experiments for each condition determined by densitometry relative to GAPDH. * $p<0.05$. Con: control; B00: bladder outlet obstruction; GAPDH: glyceraldehyde 3-phosphate dehydrogenase.

sion of AQP2-3 in the rat urinary bladder and their possible relation with NOS isoforms in a $\mathrm{BOO}$ rat model. However, we could not represent the actual functional changes in urine composition showing a water or solute movement in the urinary bladder. Further studies are needed to investigate the expression and localization of all subtypes of AQPs in the urinary bladder and their functional role in the underlying mechanisms of urinary bladder function.

\section{Conclusion}

This study showed that detrusor overactivity induced by $\mathrm{BOO}$ causes a significant increase in the expression of AQP2-3, as well as the NOS isoforms, thus providing presumptive evidence that the AQPs and NOS isoforms are involved in water and solute transport in the $\mathrm{BOO}$ rat urinary bladder.

Competing interests: None declared.
This paper has been peer-reviewed.

\section{References}

1. De Groat WC. The urothelium in overactive bladder: passive bystander or active participant? Urology 2004;64:7-11. http://dx.doi.org/10.1016/j.urology.2004.08.063

2. Johnson JA, Cavert HM, Lifson N, et al. Permeability of the bladder to water studied by means of isotropes? Am J Physiol 1951;165:87-92.

3. Nelson RA, Jones JD, Wahner HW, et al. Nitrogen metabolism in bears: urea metabolism in summer starvation and in winter sleep and role of urinary bladder in water and nitrogen conservation. Mayo Clin Proc 1975:50:141-6.

4. Negrete HO, Lavelle JP, Berg J, et al. Permeability properties of the intact mammalian bladder epithelium. Am J Physiol 1996;271:886-94.

5. Cross WR, Eardley I, Leese HJ, et al. A biomimetic tissue from cultured normal human urothelial cells: analysis of physiological function. Am J Physiol Renal Physiol 2005;289:F459-68. http://dx.doi. org/10.1152/aiprenal.00040.2005

6. Eaton $D C$. Intracellular sodium ion activity and sodium transport in rabbit urinary bladder. J Physiol 1981;316:527-44.

7. Smith PR, Mackler SA, Weiser PC, et al. Expression and localization of epithelial sodium channel in mammalian urinary bladder. Am J Physiol 1998;274:91-6.

8. Garty H, Palmer LG. Epithelial sodium channels: function, structure, and regulation. Physiol Rev 1997:77:359-96. 
Kim et al.

9. Magni F, Sarto C, Ticozzi D, et al. Proteomic knowledge of human aquaporins. Proteomics 2006;6:563749. http://dx.doi.org/10.1002/pmic.200600212

10. Hara-Chikuma $M$, Verkman AS. Aquaporin-3 functions as a glycerol transporter in mammalian skin. Biol Cell 2005;97:479-86. http://dx.doi.org/10.1042/BC20040104

11. Spector $D A$, Wade JB, Dillow $R$, et al. Expression, localization, and regulation of aquaporin-1 to -3 in rat urothelia. Am J Physiol Renal Physiol 2002;282:1034-42.

12. Rubenwolf PC, Georgopoulos NT, Clements LA, et al. Expression and Localization of Aquaporin Water Channels in Human Urothelium In Situ and In Vitro. Eur Urol 2009;56:1013-21. http://dx.doi. org/10.1016/i.eururo.2008.08.013

13. Kim SO, Song SH, Hwang EC, et al. The Expression of AQP1 and eNOS in Menopausal Rat Urinary Bladder. Int Neurourol J 2010;14:78-85. http://dx.doi.org/10.5213/inj.2010.14.2.78

14. Bouley R, Breton S, Sun T, et al. Nitric oxide and atrial natriuretic factor stimulate cGMP-dependent membrane insertion of aquaporin 2 in renal epithelial cells. J Clin Invest 2000;106:1115-26. http:// dx.doi.org/10.1172/JC19594

15. Combet $S$, Van Landschoot $M$, Moulin $P$, et al. Regulation of aquaporin-1 and nitric oxide synthase isoforms in a rat model of acute peritonitis. J Am Soc Nephrol 1999;10: 2185-2196.

16. Mumtaz FH, Khan MA, Thompson CS, et al. Nitric oxide in the lower urinary tract: physiological and pathological implications. BJU Int 2000;85:567-8.

17. Mamas AM, Reynard JM, Brading AF. Nitric oxide and the lower urinary tract: current concept, future prospects. Urology 2003;61:1079-85. http://dx.doi.org/10.1016/S0090-4295(03)00131-6

18. Ferguson DR, Kennedy I, Burton TJ. ATP is released from rabbit urinary bladder epithelial cells by hydrostatic pressure changes-a possible sensory mechanism? J Physiol 1997;505:503-11. http://dx.doi. org/10.1111/j.1469-7793.1997.503bb.x

19. Hohlbrugger $G$. The vesical blood-urine barrier: a relevant and dynamic interface between renal function and nervous bladder control. J Urol 1995;154:6-15. http://dx.doi.org/10.1016/S0022-5347(01)67208-2
20. Schmidt-Nielsen B. Excretion in mammals: role of the renal pelvis in the modification of the urinary concentration and composition. Fed Proc 1997;36:2493-503.

21. Levinsky NG, Berliner RW. Changes in composition of the urine in ureter and bladder at low urine flow. Am J Physiol 1959;196:549.

22. Ferguson DR. Urothelial function. BJU Int 1999;84:235. http://dx.doi.org/10.1046/j.1464410x.1999.00187.x

23. Achard JM, Bubien JK, Benos DJ, et al. Stretch modulates amiloride sensitivity and cation selectivity of sodium channels in human B lymphocytes. Am J Physiol 1996;270:224-34.

24. Banks, FC, Dodd PH, Laycock JF, et al. Sodium and potassium concentration in urine can influence micturition volume in humans. Clin Sci 1997;84:107.

25. Araki I, Du $S$, Kamiyama $M$ et al. Overexpression of epithelial sodium channels in epithelium of human urinary bladder with outlet obstruction. Urology 2004;64:1255-60. http://dx.doi.org/10.1016/i. urology.2004.06.064

26. Verkman AS. More than just water channels: unexpected cellular roles of aquaporins. I Cell Sci 2005;118:3225-35. http://dx.doi.org/10.1242/ics.02519

27. Braam B. Renal endothelial and macula densa NOS: integrated response to changes in extracellular fluid volume. Am J Physiol 1999;276:1551.

28. Truschel ST, Wang E, Ruiz WG et al. Stretch-regulated exocytosis/endocytosis in bladder umbrella cells. Mol Biol Cell 2002;13:830-46. http://dx.doi.org/10.1091/mbc.01-09-0435

Correspondence: Dr. Soo Bang Ryu, Department of Urology, Chonnam National University Hospital and Medical School, 8, Hak-dong, Dong-ku, Gwangiu \#501-757, South Korea; fax: +82-62-2271643; sbryu@chonnam.ac.kr 